\title{
Neurobehavioural Disorders after Traumatic Brain Injury: Assessment, Treatment and Outcome
}

The World Health Organisation has predicted that traumatic brain injury (TBI), will constitute the third largest contributor to the global burden of disease and disability (after heart disease and depression) by 2015 (Murray \& Lopez, 1997). The significance of this lies not simply in the number of cases likely to need rehabilitation and social care but also the complex pattern of disability associated with TBI, comprising abnormalities of cognition, personality, and behaviour, now referred to as neurobehavioural disability (Wood, 1987). This term was introduced into the clinical nomenclature to emphasise the point that inter-related disorders of cognition, social behaviour, emotional expression, and personality were 'organic' consequences of TBI that resulted in altered and often challenging behaviour. These characteristics need to be distinguished from emotional or behaviour disorders that primarily represent a psychological reaction to the injury experience (Wood, 1990, 2001).

Neurobehavioural disability is difficult to rehabilitate and its full psychosocial impact is usually not recognised until the post-acute stage of recovery, well after the person has been discharged from hospital. The complex pattern of problems often leads to life-long social handicap that has an impact not only on the injured person but also their family and social network (Kreutzer, et al., 1994a \& b; Morton \& Wehman, 1995).

This Anniversary Edition of Neurorehabilitation opens by outlining some of the main characteristics of neurobehavioural disability and their impact on psychosocial outcome. The next section addresses some approaches to assessing problems of social cognition that can have major psychosocial implications in the years after injury. This section also explores methods to identify obstacles to effective rehabilitation caused by avoidant coping styles, then closes by presenting the results of a systematic review of outcome measures on traumatic brain injury, using the framework of the International Classification of Functioning, Disability and Health (ICF). The second section comprises a consideration of some of the principles, practices, and challenges surrounding psychological approaches designed to address and rehabilitate those who exhibit various kinds of neurobehavioural disorders. The different contributions contrast behavioural, psychotherapeutic, and finally community-based approaches that employ technological aides to improve consistency and reduce costs of long term care/rehabilitation programmes. The final section also considers the clinical and cost effectiveness of rehabilitation, partly by examining some of the methodological issues surrounding outcome studies, partly by a consideration of different types of intervention, and partly by identifying some of the factors that can act as obstacles to psychosocial recovery.

These contributions, led by eminent clinicians, each of whom employs a science-practitioner approach to their work, will hopefully provide a frame of reference for rehabilitation practitioners to consider their approach to the life-long changes in cognition, behaviour, and personality that comprise neurobehavioural disability after traumatic brain injury, and the need for effective post-acute rehabilitation interventions, to improve long term psychosocial outcome.

Rodger Ll. Wood Guest Editor College of Human \& Health Science, Vivian Building 


\section{Swansea University \\ Swansea SA2 8PP \\ Wales, UK}

Tel.: +44 1792295778

Fax: +44 1792295009

E-mail: r.1.wood@swansea.ac.uk.

\section{References}

Kreutzer, J. S., Gervasio, A., \& Camplair, P. (1994a). Patient correlates of caregiver's distress and family functioning after traumatic brain injury. Brain Injury, 8, 211-230.

Kreutzer, J. S., Gervasio, A., \& Camplair, P. (1994b). Primary caregiver's psychological status and family functioning after traumatic brain injury. Brain Injury, 8, 197-210.
Morton, M. V., \& Wehman, P. (1995). Psychosocial and emotional sequelae of individuals with traumatic brain injury: A literature review and recommendations. Brain Inj, 9(1), 81-92.

Murray, C. J., \& Lopez, A. D. (1997). Alternative projections of mortality and disability by cause 1990-2020: Global Burden of Disease Study. Lancet, 24, 349(9064), 1498-1504.

Wood, R. L. L. (1987). Brain Injury Rehabilitation: A Neurobehavioural Approach. London: Croom Helm.

Wood, R. Ll. (1990). A neurobehavioural paradigm for brain injury rehabilitation. In R. Ll. Wood (Ed.), Neurobehavioural Sequelae of Traumatic Brain Injury (pp. 3-17). London: Taylor and Francis.

Wood, R. Ll. (2001). Understanding neurobehavioural disability. In R. L1. Wood \& T. McMillan (Eds.), Neurobehavioural Disability \& Social Handicap, after Head Injury. London: Psychology Press. 